\title{
Die Kinetik der Aufnahme von im Wasser gelöstem Sauerstoff durch die menschliche Haut
}

\author{
Von Bogislav Rackow \\ Aus dem Institut für Physikalische Chemie der Friedrich-Schiller-Universität, Jena \\ (Z. Naturforschg. 9 b, 487-492 [1954]; eingegangen am 20. Januar 1954)
}

1. Die $\mathrm{O}_{2}$-Aufnahme aus dem Wasser durch die Haut wurde mit Hilfe der „abgekürzten Polarographie“" gemessen. Die Apparatur wird beschrieben.

2. Es wurden Konzentrations-Zeitkurven aufgenommen bei verschiedener Temperatur und Durchblutung sowie Anämisierung, Amputation, Bestrahlung und Senfölreizung.

3. Die $\mathrm{O}_{2}$-Aufnahme wurde quantitativ berechnet. Aus dem Verlauf der Kurven und ihrer Abhängigkeit von den unter 2. genannten Faktoren wurde gezeigt, daß der Vorgang durch eine Uberlagerung einer Oxydation mit einer Diffusion dergestalt zustande kommt, daß die Geschwindigkeiten beider einander kommensurabel sind und bei höherer $\mathrm{O}_{2}$-Konzentration die Oxydation, bei niederer die Diffusion geschwindigkeits-bestimmend ist.

4. Die Oxydation findet bereits in den äußeren Gewebsschichten statt, ohne daß der $\mathrm{O}_{2}$ Verbrauch vom Bluttransport beeinflußt wird.

$\mathrm{D}_{\mathrm{s}}$ a die Frage, ob mit einer merklichen Geschwindigkeit und mit welcher Geschwindigkeit im Wasser gelöster $\mathrm{O}_{2}$ von der menschlichen Haut aufgenommen wird, bisher nicht geklärt war, obwohl z. B. schon $\mathrm{O}_{2}$-entwickelnde Badesalzpräparate im Handel sind, ist diese Frage nunmehr in einer Arbeit, die Verf. gemeinsam mit $\mathrm{Kihn}$ durchgeführt hat ${ }^{*}$, beantwortet worden. Die für die Innere Medizin und die Balneologie interessanten Ergebnisse werden von $\mathrm{Kihn}$ an anderer Stelle veröffentlicht. Die Ergebnisse sind an zahllosen Gesunden und Kranken jeden Alters, zu verschiedenen Zeiten des Tages, des Ovarzyklus, nach verschiedenartigen Prozeduren usw. gewonnen worden. Da jedoch diese Arbeit auch zu physikalisch-biochemischen Ergebnissen geführt hat, sollen diese hier im folgenden dargestellt werden.

\section{Die A p paratur (Abb. 1)}

Als Versuchsanordnung wurde eine ähnliche handliche Apparatur gewählt wie diejenige, mit der Petering und Daniels ${ }^{1}$ sowie Moore und Duggar ${ }^{2}$ den $\mathrm{O}_{2}$-Verbrauch und die $\mathrm{O}_{2}$-Entwicklung biologischer Reaktionen verfolgt haben, wie z. B. Gärungsvorgänge, Oxydationsprozesse im Blut oder Quantenausbeute der Photosynthese. Sie gestattet, in wenigen Augenblicken den jeweiligen $\mathrm{O}_{2}$-Gehalt zu bestimmen und ihn im Verlaufe der Zeit zu verfolgen. Das ist dadurch mög-

* Vorgetragen am 6.10. 1953 von $\mathrm{Kihn}$ vor der Gesellschaft für Balneologie in Bad Reichenhall.

1 H. G. P e t e ring u. F. D a n i e ls, J. Amer. chem. Soc. 60, 2796-2802 [1938].

2 W. E. M o ore u. B. M. D u g g a r, „Photosynthesis in Plants“ Chapt. 11, 1949. lich, daß man von der polarographischen Versuchsanordnung zwar die Quecksilbertropfelektrode, das Spiegelgalvanometer und eine Stromquelle benutzt, dagegen aber auf die graphisch aufzeichnende Maschinerie des Polarographen verzichtet. Man macht sich die Tatsache zunutze, daß das $\mathrm{O}_{2}$-Polarogramm eine Stufe bei 0,0 bis $-0,4 \mathrm{~V}$ entsprechend dem Vorgange $\mathrm{O}_{2}+\mathrm{H}_{2}=\mathrm{H}_{2} \mathrm{O}_{2}$ sowie eine zweite Stufe bei $-0,8 \mathrm{~V}$ entsprechend dem Vorgange $\mathrm{H}_{2} \mathrm{O}_{2}+2 \mathrm{H}=2 \mathrm{H}_{2} \mathrm{O}$ besitzt.

Diese beiden Stufen werden also aus dem Polarogramm abgesondert, auf das übrige Polarogramm wird verzichtet. Wenn man also an die beiden Elektroden, d. h. an die kleine polarisierbare Quecksilbertropf-Kathode

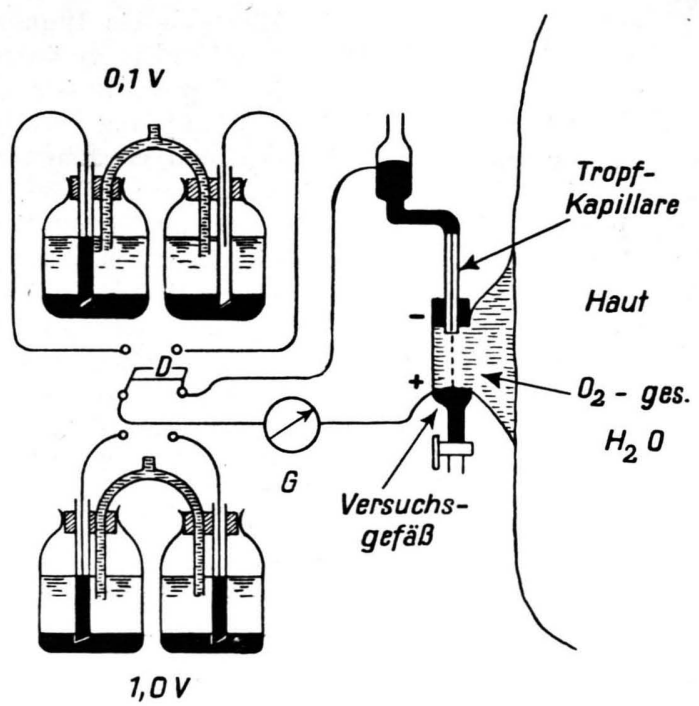

Abb. 1. Meßanordnung der „abgekürzten Polarographie“ zur $\mathrm{O}_{2}$-Bestimmung in einer an der Haut befindlichen Lösung. 
und an die große unpolarisierbare Kalomel-Quecksilberanode, zwischen denen sich $\mathrm{O}_{2}$-gesättigtes Wasser befindet, zunächst etwa $0,1 \mathrm{~V}$, dann etwa $1,0 \mathrm{~V}$ Spannung anlegt, so wird praktisch die gesamte Stromzunahme, die die Spannung von $1,0 \mathrm{~V}$ gegenüber der Spannung von $0,1 \mathrm{~V}$ aufweist, durch die depolarisierende Wirkung des $\mathrm{O}_{2}$ an der Kathode bewirkt. Denn es wird durch den Luftsauerstoff die H-Polarisation und damit die $\mathrm{Ab}$ sperrung des Stromes aufgehoben. So ist also die Höhe beider $\mathrm{O}_{2}$-Stufen, die hier die Stromzunahme zwischen $0,1 \mathrm{~V}$ und $1,0 \mathrm{~V}$ ausmachen, proportional dem $\mathrm{O}_{2}$-Gehalt. Damit das Wasser den Strom besser leitet und sich an der Kalomelanode Chlor-Ionen entladen können, haben wir auf $5 \mathrm{ccm}$ Wasser $0,1 \mathrm{ccm}$ gesättigte $\mathrm{KCl}$ Lösung hinzugefügt. Die durch die angrenzende Haut bewirkte Änderung der Salzmenge in der Lösung muß klein sein gegenüber dem eigenen Salzgehalt der Lösung, um deren Eigenwiderstand konstant zu halten. Auf eine Sättigung des Wassers mit Kalomel sowie auf Kalomel als festen Bodenkörper mußte verzichtet werden, da sonst durch die Wanderung von Quecksilberionen aus der Anode zur Kathode der sogenannte Reststrom unterhalb $0,1 \mathrm{~V}$ Zersetzungsspannung so groß geworden wäre, $\mathrm{da} ß$ dagegen die uns wichtige Stromzunahme zwischen 0,1 und $1,0 \mathrm{~V}$ klein erschienen und diese Methode zu unempfindlich geworden wäre. $\mathrm{Da} ß$ infolge des anfänglichen Fehlens von Kalomel auch die Anode, die in unserem Falle aus technischen Gründen nicht besonders groß gemacht werden konnte, im Laufe der mehrere Stdn. dauernden Versuche etwas polarisiert wurde, und wie dieses $\mathrm{zu}$ berücksichtigen war, wird noch weiter unten dargelegt.

Um von einer apparativen Vorrichtung für Spannungsstabilisierung und potentiometrischer Spannungsteilung frei zu sein, benutzten wir folgende Anordnung: Zur Erzeugung der Spannung von 0,1 V benutzten wir ein Element, welches im gesonderten mit Lösungsheber verbundenen Fläschchen als die positive Elektrode ein 10-proz. Bleiamalgam in 1-m. $\mathrm{CdJ}_{2}$-Lösung, mit $\mathrm{PbJ}_{2}$ gesättigt, und als die negative Elektrode ein 11-proz. Blei- und 9-proz. Kadmiumamalgam in 1-m. $\mathrm{CdJ}_{2}$-Lösung enthält. Die Spannung von $1,0 \mathrm{~V}$ wird von einem Weston-Normalelement bezogen, welches wieder aus zwei Flaschen besteht. In einer derselben betätigt sich als der negative $\mathrm{Pol}$ ein 13-proz. Kadmiumamalgam in einer 2-m. $\mathrm{CdSO}_{4}$ Lösung und in der zweiten als der positive Pol reines Quecksilber mit einer Merkurosulfatschicht, wiederum in einer 2-m. Cd $\mathrm{SO}_{4}$-Lösung. Diese Anordnung wurde von Petering und Daniels (s. o.) wegen ihrer Spannungskonstanz und ihres leichten Transportes in die freie Natur angegeben. Die zu untersuchende Flüssigkeit befand sich in dem mit einem Glockenansatz versehenen Elektrodengefäß. Dieses wurde an die Haut gesetzt und mit einer Gummihalterung befestigt. Dann wurde gefüllt und mit dem Stopfen, der um die Quecksilberkapillare saß, verschlossen. Etwaige unter der Glasglocke sitzende Luftblasen mußten mit einer Hohlnadel herausgesaugt werden. Dies zu beachten, erschien wichtig; denn es zeigte sich im Verlaufe der Versuche, daß jedesmal, wenn der $\mathrm{O}_{2}$-Gehalt nicht regelmäßig von einer Messung zur anderen abnahm, dies auf eine von außen in das Versuchsgefäß eingedrungene Luftblase zurückgeführt werden konnte. Es erwies sich daher als zweckmäßig, eine Abdichtung des Glockenrandes mit Collidon vorzunehmen, wodurch ein luftdichter Film erzeugt wurde. Die Kapillare war mit einem Quecksilber-Vorratsgefäß durch einen Schlauch verbunden, an diesem war die negative Stromzuführung befestigt. Der positive Pol war an das Quecksilber gelegt, welches als Bodenkörper im Elektrodengefäß lag. Die einzelnen Messungen wurden alle 12 Min. vorgenommen. Durch Heben des Vorratsgefäßes wurde vor jeder einzelnen Messung die Elektrode zum Tropfen gebracht. Zunächst wurde $0,1 \mathrm{~V}$ angelegt und der Ausschlag des Spiegelgalvanometers in Skalenteilen abgelesen. Dann wurde $1,0 \mathrm{~V}$ angelegt und abermals abgelesen. Schließlich wurde durch Senken des Vorratsgefäßes die Tropfelektrode wieder abgestellt.

Weshalb wurde diese Methode gewählt?

B a u m berge r und Mitarbb. ${ }^{3}$ bestimmten z. B. $\mathrm{O}_{2}$ in physiologischer Lösung, indem sie mit einer einzigen konstanten Spannung von $0,5 \mathrm{~V}$ arbeiteten. Diese Methode ist nur zuverlässig, wenn die Anode von der Kathode durch einen Agar-Heber getrennt ist und die Anode eine gesättigte KCl-Kalomelelektrode darstellt, die unpolarisierbar ist. Denn die Ablesung bei $0,1 \mathrm{~V}$, die von uns bei jeder Messung vorgenommen wurde, läßt den Gegenstrom erkennen, der durch die gering polarisierte Quecksilberanode entsteht, die sich im gleichen Elektrodenraum befindet. Infolgedessen ist für den $\mathrm{O}_{2}$-Gehalt nicht der Absolutwert unserer Ablesung, sondern nur die Differenz zwischen dem 0,1 V- und dem 1,0 V-Wert maßgebend, bei der die Anodenpolarisation herausfällt. Nach Beendigung unseres Gesamtversuches zeigte sich, daß die Versuchszelle selbst, d. h. ohne Anlegung der Elementspannung eine Eigenspannung anzeigte, deren Betrag sich als genau so groß erwies wie der Rückgang der Spannung, der bei der 0,1 V-Messung im Verlauf des Gesamtversuches zu verzeichnen war. Die Stärke des Reststromes hat sich also um die des entgegengesetzten Polarisationsstromes vermindert. Die Benutzung der Apparatur mit einer Kalomelanode in getrenntem Anodenraum würde die ganze an der Versuchsperson angelegte Vorrichtung sehr sperrig und schlecht sitzend machen. Das gilt in noch größerem $\mathrm{Maße}$ von den elektrochemischen $\mathrm{O}_{2}$-Bestimmungen, die ohne die Quecksilberelektroden mit festen Elektroden arbeiten. Denn in diesem $\mathrm{Fall} \mathrm{mu}$ eine durch eine mechanische Rührung erzeugte gleichmäßige Strömung an der Elektrode vorbeiziehen, und einen Rührer hier anzubringen, wäre sehr viel ungünstiger als unsere Anordnung. Daher wählten wir hier eine Elektrode, die an der Lösung vorbeiströmt, statt umgekehrt eine Lösung, die an der Elektrode vorbeiströmt.

Um uns zu vergewissern, daß die $\mathrm{O}_{2}$-Abnahme nicht durch irgendeinen unkontrollierbaren Vorgang, sondern durch die Nachbarschaft der Haut verursacht war, wurde folgender Versuch gemacht: Auf dem Oberarm der Versuchsperson wurde eine Gummihaut geklebt und auf

${ }^{3}$ J. P. Ba umberger, G. F. Leong u. K. A. B a rdwell, Federation Proc. 7, 6-7 [1948]. 
diese die Glocke befestigt. Es kam also die Haut nicht mit dem Wasser in Berührung. Dann wurde der Versuch in üblicher Weise durchgeführt. Er verlief völlig ohne Stromänderung, d.h. der $\mathrm{O}_{2}$ blieb in der Lösung erhalten, da er nicht durch das Gummi in die Haut eindringen konnte.

Eine weitere Kontrolle ergab sich aus einem anderen Versuch, bei dem ursprünglich durch künstliche Anämisierung eine Herabsetzung der Hautatmung nachgewiesen werden sollte. $\mathrm{Zu}$ diesem Zwecke wurde auf elektroendosmotischem Wege Adrenalin in die Haut eingebracht. Es resultierte aber nicht, wie erwartet, ein verminderter, sondern ein vermehrter $\mathrm{O}_{2}$-Verbrauch. Wir stellten weiter fest, daß ein in das Versuchsgefäß eingebrachter Tropfen einer Adrenalin-Lösung 1:1000 die Lösung innerhalb von $2 \mathrm{Min}$. $\mathrm{O}_{2}$-frei machte. Diese beiden Sachverhalte sind folgendermaßen zu erklären: Adrenalin enthält den Ring des Brenzkatechins mit 2 benachbarten OH-Gruppen. Brenzkatechin ist ein sehr starkes Reduktionsmittel und verbraucht $\mathrm{O}_{2}$, wobei zunächst daraus das $o$-Benzochinon-Derivat entsteht. Jedoch wurden diese Adrenalinversuche unter sorgfältiger Reinigung der Haut von Adrenalin wiederholt, es zeigte sich, daß durch die Anämisierung der Haut durch Adrenalin die Resorptionsgeschwindigkeit von $\mathrm{O}_{2}$ entweder nicht beeinflußt oder verstärkt wurde. Hierauf wird im theoretischen Teil dieser Arbeit eingegangen. Ebenso unerwartet verlief ein Versuch an einem amputierten Arm. Er nahm zunächst weiterhin $\mathrm{O}_{2}$ aus dem Wasser auf, als ob er noch lebte. Jedoch klang im Verlaufe von 10-12 Stdn. nach dem Tode diese Fähigkeit bis zum Verschwinden ab.

\section{Ergebnisse}

Ohne auf die Verschiedenheiten der Einzeluntersuchung einzugehen, gibt Abb. 2 den gefundenen Sachverhalt wieder. Es ist die $\mathrm{O}_{2}$-Konzentration $c$ des Wassers gegen die Zeit aufgetragen. $c_{\mathrm{A}}$ ist die anfängliche Sättigungskonzentration von $\mathrm{O}_{2}$ in luftgesättigtem $\mathrm{H}_{2} \mathrm{O}$ bei $22^{\circ} \mathrm{C}$ und $1 \mathrm{Atm}$. Sie beträgt $0,251 \mathrm{mMol} / l$ (d.h. ist etwa millinormal) bzw. 8,07 $\mathrm{mg} / \mathrm{l}$ bzw. $6,11 \mathrm{~cm}^{3} \mathrm{O}_{2}$-Gas (neben $\mathrm{N}_{2}$, A usw.) von $1 \mathrm{Atm}$. im Liter. $c_{\mathrm{E}}$ ist die Endkonzentration der wäßrigen $\mathrm{O}_{2}$-Lösung, die offenbar gleich der $\mathrm{O}_{2}$ Konzentration des Gewebes ist, der sich die $\mathrm{O}_{2}$-Konzentration in der Außenlösung mit der Zeit asymptotisch nähert. Sie beträgt $1 / 5$ bis $1 / 10$ von $c_{\mathrm{A}}$ und kann auch als Gleichgewichts-Konzentration bezeichnet werden. Gemessen wurde auf $6 \mathrm{~cm}^{2}$ Hautfläche und $6 \mathrm{~cm}^{3}$-Lösung. Die gemessenen Werte liegen auf Kurven, von denen 1 den schnellsten, 2 den langsamsten Verlauf wiedergibt, der von uns bei $22^{\circ} \mathrm{C}$ Zimmertemperatur und einer Hauttemperatur zwischen $33,5^{\circ}$ und $35^{\circ} \mathrm{C}$ beobachtet wurde. Alle übrigen bei diesen Temperaturen vorgenommenen Meßreihen bilden eine Kurvenschar, die zwischen 1 und 2 liegt und nicht gezeichnet ist. Nur als die Raumtemperatur statt $22^{\circ}$ nur $18^{\circ}$ und die Hauttemperatur $28,5^{\circ}$ betrug, die zu untersuchende Person fror und sich ihre Haut kalt anfühlte („Gänsehaut“), ergab diese Haut, die sonst normale Kurven zwischen 1 und 2 gab, die Kurve 3. Es wurden folgende Beobachtungen gemacht: Erstens hatten alle Kurven ohne Ausnahme denselben charakteristischen Verlauf, der die Aufmerksamkeit des Verfassers auf sich zog und der theoretischen Deutung zunächst Schwierigkeiten bereitete. Der Verlauf weist zumindest darauf hin, $\mathrm{da} ß$ es sich um keinen einfachen Vorgang handelt. Denn unerwarteterweise ist die Abnahme von $c$ mit der Zeit so lange, bis $c$ auf etwa $1 / 3$ seines Anfangswertes gesunken ist, praktisch konstant, was bei den

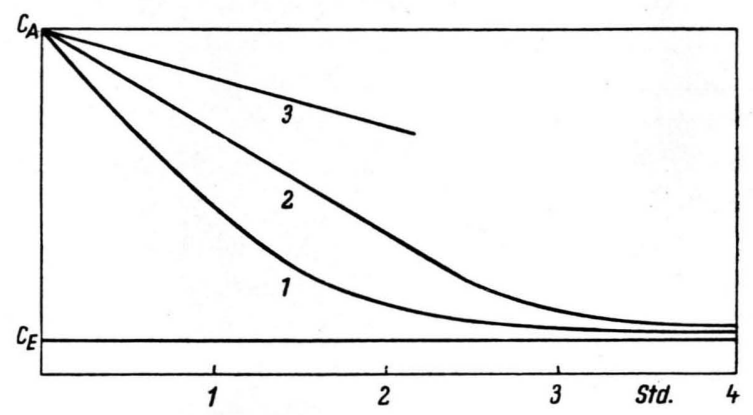

Abb. 2. Konzentrations-Zeitkurven des im Wasser gelösten $\mathrm{O}_{2}$.

schnelleren Abläufen $1^{1 / 2}$, bei den trägeren $2^{1 / 2}$ Stdn. dauert. Dann erst beginnt die Kurve sich der Horizontalen entsprechend der Endkonzentration asymptotisch anzuschmiegen. Zwischen 3 und 4 Stdn. war der Endzustand praktisch erreicht.

Zweitens fiel auf, daß der Kurvenverlauf von dem natürlichen Durchblutungszustand der zu messenden Haut vollkommen unabhängig war. Ebenso befand die Aufnahme-Geschwindigkeit sich nicht außerhalb des normalen Bereiches, wenn das Blut nur 50\% Hämoglobin (Anämie) oder 140\% Hämoglobin (Polyglobulie) enthielt, welches ja den $\mathrm{O}_{2}$-Transport besorgt.

Drittens wurde gefunden, daß die Aufnahme-Geschwindigkeit trotzdem außerordentlich stark abhängig von der Temperatur war, wie Kurve 3 zeigt.

Viertens ergab sich seltsamerweise, daß auch künstliche Beeinflussung der Blutzufuhr den $\mathrm{O}_{2}$-Verbrauch nicht beeinflußte. Wenn nämlich auf der Haut eine größere Fläche durch Adrenalin von der Blutzufuhr abgedrosselt wurde, und zwar durch einen endosmotisch erzeugten, bleichen anämischen Haut- 
bezirk, so hat dieser weiße Bezirk den gleichen $\mathrm{O}_{2}$ Verbrauch wie die ihn umgebende normale rötliche Haut. Ebenso bewirkte der umgekehrte Versuch, ein durch Blutstau hyperämisierter Arm, keine Änderung gegenüber dem normalen Arm, d. h. passive Hyperämie blieb ohne Effekt auf die $\mathrm{O}_{2}$-Aufnahme.

Fünftens war der Verlauf der $\mathrm{O}_{2}$-Aufnahme am amputierten Arm, solange es sich um Messungen im sofortigen Anschluß an die Amputation handelt und der Arm noch seine warme Temperatur hatte, unabhängig von der Abtrennung von Blutkreislauf und Nervensystem.

Sechstens war dagegen bei nervengelähmten Extremitäten mit daraus resultierender Gewebeatrophie die $\mathrm{O}_{2}$-Aufnahme bis um $1 / 3$ gegenüber den gesunden Extremitäten derselben Person herabgesetzt.

Siebentens wurde dagegen gefunden, daß ein erhöhter $\mathrm{O}_{2}$-Verbrauch durch starke Ultraviolett-, Wärmestrahlen- und Senföleinwirkung bewirkt wurde, die ja einen Angriff auf die äußeren Hautschichten darstellen.

Soweit das experimentelle Material. Diese widerspruchsvoll erscheinenden Ergebnisse, d.h. der fast knickhafte Verlauf der Kurven, die Unabhängigkeit vom natürlichen Durchblutungszustand und Hämoglobingehalt, die hohe Temperaturabhängigkeit, die Unabhängigkeit von Adrenalin-, Anämisierung- und Stauungshyperämisierung und der Amputation, die Verlangsamung durch Nervenlähmung (Atrophie des Gewebes) sowie die Beschleunigung durch angreifende physikalische und chemische Agentien machten eine im folgenden dargestellte kinetische Analyse notwendig, die dann zu einer vollständigen Klärung des Gesamtvorganges einschließlich dieser genannten Probleme führte.

\section{Theoretischer Teil}

Da die verwendete Wasserglocke den Inhalt $I$ $=6 \mathrm{~cm}^{3}$ enthielt und $F=6 \mathrm{~cm}^{2}$ Fläche (ohne Rand gerechnet) bedeckte, so gibt unsere Darstellung die Verhältnisse wieder, wie sie bei $1 \mathrm{~cm}^{3} \mathrm{H}_{2} \mathrm{O}$ auf $1 \mathrm{~cm}^{2}$ Fläche oder bei $1 \mathrm{~cm}$ dickem Wassermantel auf beliebiger Hautfläche herrschen.

Eine Kurvenschar mit einem Verlauf, wie ihn Abb. 2 zeigt, ergibt sich immer dann, wenn ein Ausgleich zwischen zwei Konzentrationen durch Diffusion oder zwei Temperaturen durch Wärmeleitung derart stattfindet, daß ein Verbrauch an chemischer Substanz bzw. Wärme gleichzeitig vorhanden ist. Ohne diesen Verbrauch wäre die Kurvenschar einfach logarithmisch. Wir rechnen hier mit dem nahezu geradlinigen Abfall der Kurve. Dieser ergibt pro Stde. im Falle der ersten Kurve $0,004 \mathrm{mg} / \mathrm{cm}^{2}$ bzw. $0,00012 \mathrm{mMol} / \mathrm{cm}^{2}$ bzw. $0,003 \mathrm{~cm}^{3} \quad \mathrm{O}_{2}$-Gas von $1 \mathrm{Atm}$. pro $\mathrm{cm}^{2}$ Hautfläche. Das sind auf einer mittleren Körperoberfläche von $2 \mathrm{~m}^{2}$ bei der ersten Kurve $80 \mathrm{mg}$ bzw. $2,4 \mathrm{mMol}$ bzw. $60 \mathrm{~cm}^{3}$ pro Stde., bei der zweiten Kurve etwa $2 / 5$ der Werte der ersten Kurve und analog bei der dritten Kurve etwa $1 / 4$ der Werte der ersten Kurve. Wie gesagt, gelten diese Angaben nur so lange, wie entweder das Wasser an $\mathrm{O}_{2}$ gesättigt ist oder noch über $1 / 3$ der Sättigungskonzentration enthält. Dieser Sachverhalt sowie die außerordentlich starke Temperaturabhängigkeit des Kurvenabfalls und dessen Unabhängigkeit von der Durchblutung sowie die übrigen experimentellen Befunde nötigen zu folgenden Schlüssen:

Dieser Vorgang ist nicht vergleichbar mit einem gewöhnlichen Konzentrations-Austausch zwischen zwei flüssigen, durch eine Membran getrennten Phasen, d. h. nicht mit einer reinen Diffusion. Denn es ist für die gleichmäßige Versorgung eines größeren Zellverbandes mit gelöstem $\mathrm{O}_{2}$ eine Selbstverständlichkeit, daß die Diffusions-Geschwindigkeit und die Verbrennungs-Geschwindigkeit so durch den Organismus einreguliert sind, daß beide einander kommensurabel sind und sich in gleicher Größenordnung befinden. Denn wäre die Verbrennungs-Geschwindigkeit zu groß, würde die Diffusion nicht weit genug in den Zellverband eindringen, um alle Zellen gleichmäßig mit $\mathrm{O}_{2}$ zu versorgen, sondern er würde von den ersten Zellen weggefangen. Wäre die Verbrennungs-Geschwindigkeit zu klein, so wäre die Ausnutzung zu gering. Also beim Einwandern wird der $\mathrm{O}_{2}$ mit einer der Einwanderungs-Geschwindigkeit vergleichbaren Geschwindigkeit schon in dem Gewebe verbrannt. Nun ist es ein bekanntes Gesetz der Reaktionskinetik, daß derjenige Teilprozeß die Geschwindigkeit des Gesamtprozesses bestimmt, der der langsamste ist. Das bedeutet, daß der Gesamtprozeß immer die Geschwindigkeit des jeweils langsamsten Teilprozesses hat. Ein anderes bekanntes Gesetz der Reaktionskinetik besagt, daß die Reaktions-Geschwindigkeit von der Konzentration der Reaktions-Teilnehmer unabhängig ist, wenn die Reaktion über eine konstante Menge eines heterogenen Katalysators verläuft und sämtliche aktiven Zentren des Katalysators von Reaktions-Teilnehmern voll besetzt sind. Man spricht von einer Reaktion „nullter Ordnung". Solange der nun hier vorliegende Oxydationsvorgang infolge vollständiger Besetzung eines 
Atmungsfermentes durch $\mathrm{O}_{2}$ von der $\mathrm{O}_{2}$-Konzentration in Lösung unabhängig ist, verläuft die Oxydation mit konstanter Geschwindigkeit, auch wenn ein Úberangebot an $\mathrm{O}_{2}$ im Gewebe zur Verfügung steht. Wenn also die Diffusion von $\mathrm{O}_{2}$ und die dadurch bedingte Beladung des Katalysators relativ schneller verläuft als die $\mathrm{O}_{2}$-Wegnahme am Katalysator durch die Zelloxydation, so bleibt nicht nur der Katalysator voll besetzt, sondern auch die $\mathrm{O}_{2}$-Nachdiffusion aus der Außenlösung muß mit derselben konstanten, konzentrations-unabhängigen Geschwindigkeit erfolgen, wie $\mathrm{O}_{2}$ im Innern wegoxydiert wird. Mit dieser Geschwindigkeit nimmt also auch der $\mathrm{O}_{2}$-Gehalt der Lösung entsprechend dem linearen Teil der Kurve von Abb. 2 ab. Ist nun aber die $\mathrm{O}_{2}$-Konzentration der Lösung so weit gesunken, daß die Geschwindigkeit, mit der die Zelloxydation den $\mathrm{O}_{2}$ vom Katalysator wegnimmt, größer ist als die EinwanderungsGeschwindigkeit, so wird der Katalysator nicht mehr voll besetzt, und es muß die Oxydation mit der immer langsamer werdenden Einwanderung Schritt halten. Nunmehr bestimmt die sich proportional der $\mathrm{O}_{2}$-Konzentration der Lösung verhaltende DiffusionsGeschwindigkeit den logarithmischen Teil der $\mathrm{O}_{2}$ Abnahme, bis der $\mathrm{O}_{2}$-Gehalt der Lösung der inneren, stationären $\mathrm{O}_{2}$-Tension gleich ist und der Prozeß zum Stillstand kommt.

Diese Vorstellung, die die Unterwasser-Hautatmung als unmittelbare Überlagerung einer Diffusion durch eine Oxydation darstellt, wobei bei höherer $\mathrm{O}_{2}$-Konzentration die Oxydations-Geschwindigkeit, bei niedrigerer die Diffusions-Geschwindigkeit den Vorgang bestimmt, wird auch durch die starke Temperatur-Abhängigkeit des Kurven-Abfalls gefordert. Eine chemische Reaktions-Geschwindigkeit in Lösung wird in der Regel in diesem mittleren Temperaturbereich bei Erhöhung um $10^{\circ}$ verdoppelt bis verdreifacht, während eine Diffusions-Geschwindigkeit sich nur um einen geringen Bruchteil erhöht. Wenn nun durch das Frieren der Haut deren Temperatur von einer Oberflächen-Temperatur von $35^{\circ}$ auf $28,5^{\circ} \mathrm{C}$ absinkt und dadurch die $\mathrm{O}_{2}$-Aufnahme auf die Hälfte reduziert wird, so ist das nicht durch die Diffusion, sondern durch die Oxydation bedingt. Die Kälteeinwirkung bewirkt eine Gefäßkontraktion und Verhinderung der Zufuhr frischen und Wegnahme verbrauchten Blutes. Dasselbe bewirkt auch Adrenalin und ganz besonders die Amputation: Jedoch wirken Adrenalin und Amputation nicht verlangsamend auf den $\mathrm{O}_{2}$-Verbrauch, der also nur auf Kosten des oxydablen Vorrats in den Hautzellen von- statten geht, solange dieser Vorrat reicht und solange die Temperatur aufrechterhalten wird. Bei Fehlen einer zentralen Zufuhr $\mathrm{O}_{2}$-reichen Blutes in die Haut wird bei Adrenalin-Anämisierung auch kein $\mathrm{O}_{2}$ von innen in die Haut transportiert, weshalb die Haut auf eine ungeschwächte Oxydation von außen angewiesen ist und dort ihren Bedarf deckt. In diesem Falle verlief ja auch die $\mathrm{O}_{2}$-Aufnahme normal und ungehindert. Eine Autoxydation des Adrenalins, wie sie an der freien Luft oder in $\mathrm{O}_{2}$-gesättigtem Wasser stattfindet, ist im Körper nicht zu erwarten, weil derartige oxydable Verbindungen durch Adsorption oder Bindung an das Eiweiß im allgemeinen vor der Autoxydation geschützt sind und im Plasma ein reduzierendes Milieu herrscht. Aus den genannten Gründen hat also die Stockung der Durchblutung durch Adrenalin keine Wirkung, im Gegensatz zur Stockung durch Kälte in bezug auf die $\mathrm{O}_{2}$-Zufuhr. Ein weiterer Beweis dafür, daß der hineindiffundierte $\mathrm{O}_{2}$ durch die intrazellulare Oxydation verbraucht wird und nicht in den Blutkreislauf geht, ist die Tatsache, daß eine rötlich durchschimmernde, sog. vollblütige Haut keine stärkere $\mathrm{O}_{2}$-Abnahme bewirkt als eine blutleer und schlaff erscheinende Haut sowie die Unabhängigkeit von Hämoglobingehalt des Blutes und der Hyperämie durch Blutstauung. Wenn angesichts der Mehraufnahme an $\mathrm{O}_{2}$ durch warme Haut das Blut am Transport des $\mathrm{O}_{2}$ beteiligt wäre, so würde das bedeuten, daß $\mathrm{O}_{2}$ aus der Haut in die Lunge transportiert werden würde, was unrationell und unwahrscheinlich wäre. Dagegen müssen physikalische und chemische Agentien, wie intensive Strahlen und Senföl, eine Erhöhung der Oxydations-Geschwindigkeit bewirken, da diese einen störenden Eingriff in das Zellplasma verursachen, zu dessen Regeneration die Zellen eine erhöhte Mobilisierung des Stoffwechsels vornehmen, die mehr $\mathrm{O}_{2}$ verbraucht, wodurch außerdem eine reaktions-beschleunigende Temperaturerhöhung (künstliches Fieber in der Haut) bewirkt wird. Andererseits muß ein atrophes Gewebe, welches infolge Nervenlähmung einen zu langsamen Stoffwechsel mit Unterernährung zeigt, naturgemäß einen langsameren $\mathrm{O}_{2}$-Verbrauch haben. So also gibt die kinetische Analyse des Gesamtvorganges eine Erklärung für alle beobachteten Erscheinungen, die, wenn man das experimentelle Material betrachtet, sowohl einander als auch unseren herkömmlichen Anschauungen und Erwartungen zu widersprechen schienen.

Auf die beschriebene Weise konnte also mit Hilfe der „abgekürzten Polarographie“ nachgewiesen wer- 
den, daß die menschliche Haut aus dem Wasser $\mathrm{O}_{2}$ aufnimmt. Es konnte ferner die Menge des aufgenommenen $\mathrm{O}_{2}$ in einer bestimmten Zeit festgestellt werden. Drittens konnte der zeitliche Verlauf der Konzentrations-Aufnahme verfolgt werden. Viertens ließ sich durch die kinetische Analyse der Konzentrations-Zeitkurve und deren Temperatur-Abhängig- keit und Unabhängigkeit von der Durchblutung u. a. die Überlagerung einer Diffusion mit einer gleichzeitigen Oxydation erkennen, wobei die OxydationsGeschwindigkeit erst dann abfällt, wenn die Kurve ihre Krümmung macht bei einer Konzentration, die die Diffusion zu langsam für die Oxydation werden läßt.

\title{
Der Alternsgang des Bindegewebes in menschlichen Organen
}

\author{
(Herz und Leber)
}

\author{
Von Rudolf Ehrenberg, Heinz Georg Winnecken und Hildegard Biebricher \\ Aus dem Physiologischen Institut der Universität Göttingen \\ (Z. Naturforschg. 9 b, 492-495[1954]; eingegangen am 26. Januar 1954)
}

\begin{abstract}
1. Es wurden zwei Verfahren entwickelt, um in gefärbten mikroskopischen Schnitten den Bindegewebsanteil der Organgewebe zu bestimmen.

2. Von 131 menschlichen Leichen im Alter von 0 bis 87 Jahren wurde in Herz und Leber der Bindegewebsanteil mit den beschriebenen Methoden bestimmt, an Hautschnitten wurde die Bindegewebsschicht direkt gemessen.

3. Wie aus den Abb. 1 bis 5 ersichtlich ist, nimmt der Gehalt an Bindegewebe in den untersuchten Organen von Anbeginn an kontinuierlich und mit gleichbleibender Steilheit des Anstiegs zu.

4. Es wird erörtert, welchen biologischen Sinn die Bindegewebszunahme haben kann, sie charakterisiert sich als echten Alternsgang (Biorheuse) in Richtung auf die Verfestigung (Erstarrung) der Organgewebe.
\end{abstract}

$\mathrm{E}$ ne Zunahme des Bindegewebsanteils der Organe und Gewebe im höheren Alter ist bekannt und wird als „Altersfibrose“ diesem Lebensabschnitt zugerechnet. Ausgehend von der Anschauung, daß der Vorgang des Alterns nicht nur eine Erscheinung sei, welche eine spätere Lebensepoche von den voraufgehenden unterscheide, sondern daß er den ganzen Lebensablauf - „Biorheuse“ — begleite, ja ihn recht eigentlich darstelle, stellten wir uns die Aufgabe, zu untersuchen, ob auch die Bindegewebszunahme einen solchen kontinuierlichen Alternsgang erkennen lasse.

Auf die in der Literatur niedergelegten experimentellen Begründungen zur Biorheuse-Theorie soll hier nicht eingegangen werden, besonders die zahlreichen Untersuchungen, die Max Bürger mit seinen Mitarbb. durchgeführt hat, haben ein reiches Material in dieser Hinsicht geliefert; wir beschränken uns auf die Darstellung unserer Ergebnisse.

\section{Methodik}

Bei der Entwicklung eines Verfahrens zur Bestimmung des Bindegewebsanteils in Organen und
Geweben mußte von der Überlegung ausgegangen werden, daß unsere Problemstellung die Verarbeitung eines umfangreichen Materials erforderte. Jede der in Jahrzehnten zusammengefaßten Altersgruppen mußte eine ausreichende Anzahl von Individuen enthalten, um von Gruppe zu Gruppe vergleichbare Werte zu ergeben. Damit entfielen alle zeitraubenden chemisch-analytischen Methoden, und die Aufgabe präzisierte sich dahin, im gefärbten mikroskopischen Schnitt den Bindegewebsanteil zu bestimmen. Eine 100-proz. selektive Bindegewebsfärbung existiert noch nicht, wohl aber gibt es Farblösungen, in denen das Bindegewebe bevorzugt angefärbt wird. Unter den 6 von uns erprobten Färbungsmethoden erwies sich die bekannte van GiesonFärbung als die für unsere Zwecke brauchbarste, da sie in der Handhabung einfach und wenig zeitraubend ist und keine der anderen Methoden ihr gegenüber entschiedene Vorzüge erkennen ließ.

Wir verarbeiteten Organmaterial aus dem Pathologischen Institut, und zwar wählten wir als erste Organe Herz und Leber als zwei Organe von strukturell und funktionell sehr verschiedener Art. Die 